\title{
Quercetin- A Mini Review
}

\author{
Muhammad FM ${ }^{1}$, Ahsan $\mathbf{M}^{2}$ and Abdul $\mathrm{W}^{3}$ \\ ${ }^{1}$ South China University of Technology, Guangzhou, China \\ ${ }^{2}$ Department of Chemistry, Government College University Faisalabad, Pakistan \\ ${ }^{3}$ Government College University Faisalabad-38000, Pakistan
}

*Corresponding author: Muhammad Faisal Manzoor, School of Food Science and Engineering, South China University of Technology, Guangzhou, China

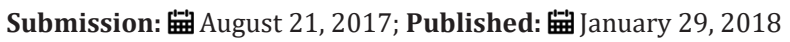

\begin{abstract}
Quercetin a flavonoid to be a member of a group of flavonols is universally consumed in the human diet and responsibly to give the color to various fruits and vegetables [1]. Mainly occur in the form of glycosides, but their other derivative have been identified [2-5]. This paper presents some recent advances regarding quercetin structure, derivatives, sources and their medicinal benefits with their effective dosage [6-8].

Keywords: Quercetin; Derivatives; Plant sources; Medicinal benefits

Abbreviations: Fe-NTA: Ferric Nitrilo Triacetate; MIC: Minimum Inhibitory Concentration, NF- KB; Nuclear Factor Kappa B, MBC: Minimum Bactericidal Concentration, INOS: Inducible Nitric Oxide Synthase
\end{abstract}

\section{Introduction}

It is a 3, 5, 7, 3, 4-pentahydroxyflavon having the five hydroxyl groups placed at five different positions. [9] The chemical structure and different derivatives of quercetin presents in different food sources such as, Mango, plums, cranberry, blueberry, chokeberry, Buckwheat, honey, beans, lettuce, chicory, onion in different

amount. Quercetin 3-0-galactoside, Quercetin 3-O-glucoside, Quercetin 3-0-glucuronide, Quercetin 7-0-glucoside, Quercetin 3-0-diglucoside and Quercetin 3-methyl ether are some derivatives that have been discovered in different plants [10-12] (Table $1 \&$ Figure 1).

Table 1: Some important derivatives of quercetin and their food sources.

\begin{tabular}{|c|c|c|c|c|}
\hline \multirow{2}{*}{ Derivatives } & \multirow{2}{*}{ Food Sources } & \multicolumn{2}{|c|}{ Contents (Mg/Kg) } & \multirow{2}{*}{ References } \\
\hline & & d.m. & f.m. & \\
\hline \multirow{6}{*}{ Quercetin 3-0-galactoside } & Mango & $75-1469$ & & [1] \\
\hline & Plums & & $\sim 36$ & {$[2]$} \\
\hline & Blueberry & & $145-146$ & [3] \\
\hline & Cranberry & & $96-97$ & \\
\hline & Chokeberry & & $414-415$ & \\
\hline & Lingon berry & & $117-116$ & \\
\hline \multirow{4}{*}{ Quercetin 3-0-glucoside } & Mango & $78-1046$ & & [1] \\
\hline & Beans & $101-692$ & & [4] \\
\hline & Plums & & 23-Nov & {$[2]$} \\
\hline & Onions & & Oct-38 & [5] \\
\hline \multirow{7}{*}{$\begin{array}{l}\text { Quercetin 3-0-xyloside } \\
\text { Quercetin 3-0-rutinoside (rutin) }\end{array}$} & Mango & $11-279$ & & [1] \\
\hline & Plums & & $27-78$ & [2] \\
\hline & Cherries & & $17-136$ & [6] \\
\hline & Tomatoes & & $3.21-9.22$ & [7] \\
\hline & Buckwheat -leave & $34-97 \times 10^{3}$ & $11-50 \times 10^{3}$ & [8] \\
\hline & Buckwheat-seeds & $441-512$ & & [9] \\
\hline & Chokeberry & & $710-711$ & [7] \\
\hline
\end{tabular}




\begin{tabular}{|c|c|c|c|c|}
\hline Quercetin 3-0-diglucoside & Beans & $122-641$ & & [4] \\
\hline Quercetin 3,3'-dimethyl ether & Honey & & $0.3-2.1$ & [10] \\
\hline \multirow{2}{*}{ Quercetin 3-0-glucuronide } & Lettuce & $0-732$ & & [11] \\
\hline & Chicory & & $80-1064$ & [12] \\
\hline $\begin{array}{l}\text { Quercetin 3-0-6"- } \\
\text { acetylglucoside }\end{array}$ & Beans & Nov-52 & & [4] \\
\hline $\begin{array}{l}\text { Quercetin 3-0-6"- } \\
\text { acetylglucoside }\end{array}$ & Beans & Nov-51 & & [4] \\
\hline Quercetin 3-methyl ether & Honey & & $2-3.34$ & [10] \\
\hline \multirow{4}{*}{ Quercetin 3-0-rhamnoside } & Mango - fruits & $0-118$ & & [1] \\
\hline & Pepper - fruits & $112-991$ & & [13] \\
\hline & Cranberry & & $54-55$ & {$[3]$} \\
\hline & Lingon berry & & $108-109$ & \\
\hline Quercetin 7-0-glucoside & Beans & $21-121$ & & [4] \\
\hline Quercetin 3,4'-diglucoside & Onions & & $168-1373$ & [5] \\
\hline
\end{tabular}

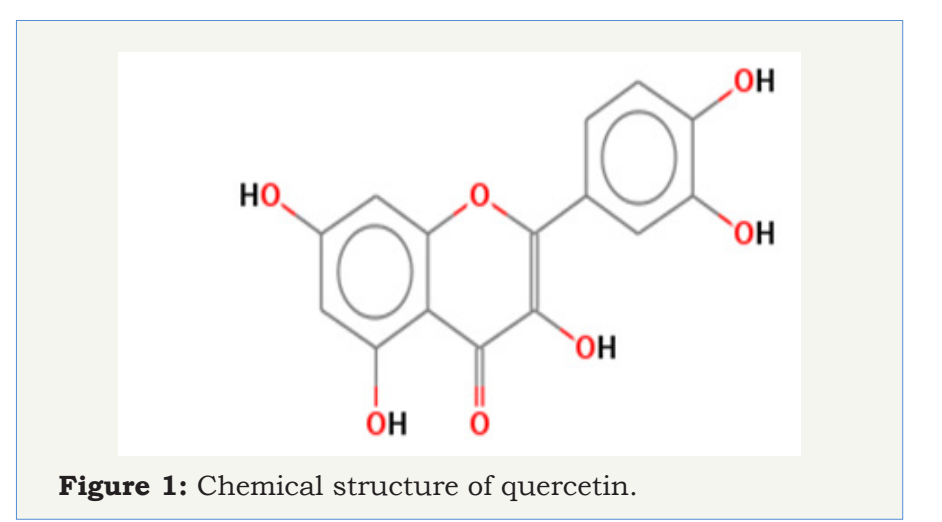

Quercetin is one of the most abundant food based flavonoids present in various edible vegetables, fruit and wine [13]. Quercetin contents present in different plants sources are categorized in different categories such as, vegetable, fruit, plant, herb, shrub, beverage and others [14-16]. The most prominent sources of quercetin is canned capers $180.76 \mathrm{mg} / 100 \mathrm{~g}$ (Table 2). It has different medicinal benefits against brain disorder, renal injury, cardiovascular diseases, blood pressure, cancer, bacterial activity, inflammation, diabetes mellitus, arthritis and asthma [17-19]. Medicinal benefits of quercetin with effective dose concentrations presented in Table 3 [20-22].

Table 2: Various food sources and quercetin contents (mg/100g).

\begin{tabular}{|c|c|c|c|c|c|}
\hline \multicolumn{2}{|c|}{ Food Sources } & \multirow{2}{*}{$\begin{array}{c}\text { Quercetin contents } \\
27.61\end{array}$} & \multicolumn{2}{|c|}{ Food Sources } & \multirow{2}{*}{$\begin{array}{c}\text { Quercetin contents } \\
2.01\end{array}$} \\
\hline \multirow{15}{*}{ Vegetables } & Ancho pepper & & \multirow{15}{*}{ Fruits } & Unsweetened apple sauce & \\
\hline & Raw broccoli & 3.22 & & Raw apple with skin & 4.43 \\
\hline & Cooked broccoli & 1.07 & & Raw apricot & 2.54 \\
\hline & Raw celery & 3.51 & & Raw bilberry & 3.05 \\
\hline & Canned green bean & 1.5 & & Black grapes & 2.56 \\
\hline & Raw green beans & 2.74 & & Frozen blueberry & 3.94 \\
\hline & Hot green raw chili pepper & 16.81 & & Raw blueberry & 3.12 \\
\hline & Hot wax raw yellow pepper & 50.62 & & Raw cherry & 1.24 \\
\hline & Iceberg lettuce & 2.48 & & Raw cowberry & 21.01 \\
\hline & Raw jalapeno & 5.08 & & Canned sweet cherry & 3.21 \\
\hline & Canned kale & 4.51 & & Cherry raw tomato & 2.78 \\
\hline & Raw kale & 7.72 & & Raw cranberry & 14.01 \\
\hline & Loose-leaf lettuce & 1.94 & & Raw cranberry juice & 16.42 \\
\hline & Boiled onions & 19.37 & & Frozen chokeberry & 8.91 \\
\hline & Red raw onion & 19.94 & & Raw lemon without peel & 2.3 \\
\hline
\end{tabular}




\begin{tabular}{|c|c|c|c|c|}
\hline Serrano raw pepper & 15.96 & & Lingo raw berry & 12.17 \\
\hline Raw spinach & 4.87 & & Lingo berry juice & 1.03 \\
\hline Spring raw onion & 14.23 & & Raw plums & 1.21 \\
\hline Sweet potato raw leave & 20.55 & & Red raw currant & 0.96 \\
\hline Raw watercress & 4.01 & & Red grapes & 3.55 \\
\hline White sweet raw onion & 5.2 & & Frozen rowanberry & 7.41 \\
\hline Yellow snap raw beans & 3.01 & & White raw currant & 1.96 \\
\hline Bog wild frozen whortleberries & 17.71 & \multirow{3}{*}{$\begin{array}{l}\text { Herbs \& } \\
\text { shurbs }\end{array}$} & Dill fresh weed & 55.13 \\
\hline Buckwheat & 23.08 & & Queen Anne's Lace leave & 1.11 \\
\hline Buckwheat flour & 2.71 & & $\begin{array}{c}\text { European raw black } \\
\text { currant }\end{array}$ & 5.7 \\
\hline Butterhead lettuce & 1.2 & \multirow{5}{*}{ Beverages } & Black brewed tea & 2.08 \\
\hline Canned capers & 180.76 & & Decaf brewed tea & 2.85 \\
\hline Raw chives & 4.76 & & Decaf green brewed tea & 2.76 \\
\hline Dry unsweetened cocoa powder & 20.14 & & Green brewed tea & 2.7 \\
\hline Raw coriander & 5.01 & & Tomato canned juice & 1.47 \\
\hline Corn poppy leave & 26.31 & \multirow{3}{*}{ Others } & Greek greens pie & 12.41 \\
\hline Raw dock leave & 86.24 & & Marinara sauce & 0.92 \\
\hline Raw lovage leave & 170.01 & & Canned tomato puree & 4.13 \\
\hline
\end{tabular}

Table 3: Medicinal benefits of quercetin with effective dose concentrations.

\begin{tabular}{|c|c|c|c|c|}
\hline \multirow{13}{*}{$\begin{array}{l}\text { Medicinal benefits } \\
\text { of quercetin }\end{array}$} & Disease & Effective & Dosage & References \\
\hline & \multirow{3}{*}{ Neuron protective } & Reverse the neurotoxicity in mice brain induced by d-galactose & 5 and $10 \mathrm{mg} / \mathrm{kg}$ & {$[14]$} \\
\hline & & $\begin{array}{l}\text { To protect the brain from the oxidative stress because act as a } \\
\text { powerful antioxidant }\end{array}$ & $100 \mathrm{mg} / \mathrm{kg}$ & [15] \\
\hline & & $\begin{array}{c}\text { Play an important against memory defects such as } \\
\text { Alzheimer's disease and cognitive impairment induced by } \\
\text { lipopolysaccharide }\end{array}$ & $25-100 \mathrm{mg} / \mathrm{kg}$ & [16] \\
\hline & Renal injury & $\begin{array}{l}\text { Diminished (Fe-NTA) ferric nitrile tri acetate oxidative renal } \\
\text { injury }\end{array}$ & $2 \mathrm{mg} / \mathrm{kg}$ & [17] \\
\hline & \multirow[t]{2}{*}{ Cardiovascular } & $\begin{array}{l}\text { Its modulate the production of nitric oxide and endothelin- } 1 \text {, } \\
\text { thereby improve endothelial function and ultimately lead } \\
\text { toward the beneficial Cardiovascular effects }\end{array}$ & $200 \mathrm{mg}$ & \multirow[t]{2}{*}{ [19] } \\
\hline & & Very effective to reduce HDL cholesterol significantly & $500 \mathrm{mg}$ & \\
\hline & Blood Pressure & Suppress the blood pressure in hypertensive patients & $730 \mathrm{mg}$ & {$[20]$} \\
\hline & Cancer & $\begin{array}{l}\text { Very effective in breast adenocarcinoma and inhibit the tumor } \\
\text { growth }\end{array}$ & $1 \mathrm{mg} / \mathrm{kg}$ & {$[21]$} \\
\hline & \multirow{4}{*}{ Antibacterial activity } & Inhibitory effects on Streptococcus mutans & $\begin{array}{l}\mathrm{MIC}(2 \mathrm{mg} / \mathrm{ml}) \\
\mathrm{MBC}(8 \mathrm{mg} / \mathrm{ml})\end{array}$ & \multirow{4}{*}[22]{} \\
\hline & & $\begin{array}{l}\text { Inhibitory effects on Streptococcus sobrinus and } \\
\text { Aggregatibacter actinomycetemcomitans }\end{array}$ & $\begin{array}{l}\mathrm{MIC}(1 \mathrm{mg} / \mathrm{ml}) \\
\mathrm{MBC}(8 \mathrm{mg} / \mathrm{ml})\end{array}$ & \\
\hline & & $\begin{array}{l}\text { Inhibitory effects on Lactobacillus acidophilus and } \\
\text { Streptococcus sanguinis }\end{array}$ & $\begin{array}{l}\text { MIC }(2 \mathrm{mg} / \mathrm{ml}) \\
\text { MBC }(16 \mathrm{mg} / \mathrm{ml})\end{array}$ & \\
\hline & & Inhibitory effects on Prevotella intermedia & $\begin{array}{l}\text { MIC }(4 \mathrm{mg} / \mathrm{ml}) \\
\text { MBC }(16 \mathrm{mg} / \mathrm{ml})\end{array}$ & \\
\hline
\end{tabular}




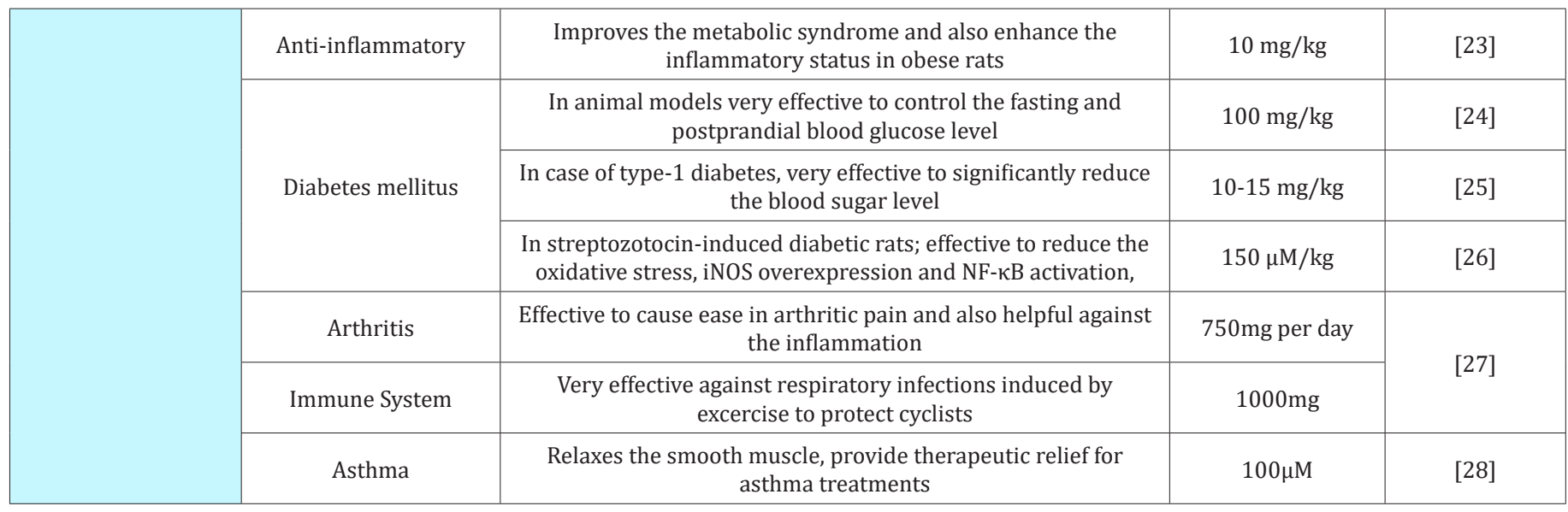

\section{Conclusion}

Quercetin generally exist in the edible plants and mostly used to manufacturing of traditional medicine to relieve some type of diseases [23-25]. In some recent studies quercetin exerts its biological properties in vivo. Overall, the best source of quercetin is canned carpers with $180.76 \mathrm{mg} / 100 \mathrm{~g}$ and followed by raw lovage leave with $170.01 \mathrm{mg} / 100 \mathrm{~g}$ [26-28].

\section{References}

1. Berardini N, Fezer R, Conrad J, Beifuss U, Carle R, et al. (2005) Screening of mango (Mangiferaindica L.) cultivars for their contents of flavonol Oand xanthone C-glycosides, anthocyanin's and pectin. J Agric Food Chem 53(5): 1563-1570.

2. Kim DO, Chun OK, Kim YJ, Moon HY, Lee CY (2003) Quantification of polyphenolics and their antioxidant capacity in fresh plums. J Agric Food Chem 51(22): 6509-6515.

3. Zeng W, Wang SY (2003) Oxygen radical absorbing capacity of phenolics in blueberries, cranberries, chokenberries and lingonberries. J Agric Food Chem 51(2): 502-509.

4. Chang Q, Wong YS (2004) Identification of flavonoids in Hakmeitau beans (Vignasinensis) by high performance liquid chromatog-raphyelectrospray mass spectrometry (LC-ESI/MS). J Agric Food Chem 52(22): 6694-6699.

5. Nemeth K, Piskuła MK (2007) Food content, processing, absorption and metabolism of onion flavonoids. Crit Rev Food Sci Nutr 47(4): 397-409.

6. Goncalves K, Landbo AK, Knudsen D, Silva AP, Moutinho PJ, et al. (2004) Effect of ripeness and posthar-vest storage on the phenolic profiles of cherries (Prunusavium L.). J Agric Food Chem 52(3): 523-530.

7. Slimestad R, Torskangerpoll K, Nateland HS, Johannessen T, Giske NH (2005) Flavonoids from black chokeberries, Aroniamelano-carpa. J Food Comp Anal 18: 61-68.

8. Kalinova J, Triska J, Vrchotova N (2006) Distribution of vitamin E, squalene, epicatechin and rutin in common buckwheat plants (FagopyrumesculentumMoech). J Agric Food Chem 54(15): 5330-5335.

9. Oomah BD, Mazza G (1996) Flavonoids and antioxidative activities in buckwheat. J Agric Food Chem 44(7): 1746-1750.

10. Yao L, Datta N, Tomas BFA, Ferreres F, Martos I, et al. (2003) Flavonoids, phenolic acids and abscisic acid in Australian and New ZelandLeptospermum honeys. Food Chem 81: 159-168.

11. Nicolle C, Carnat A, Fraisse D, Lamaison JL, Rock E, et al. (2004) Characterisation and variation of antioxidant micronutrients in lettuce (Lactucasativafolium). Science of Food and Agriculture 84(15): 20612069.
12. Innocenti M, Gallori S, Giaccherini C, Ieri F, Vincieri FF, et al. (2005) Evaluation of the phenolic content in the aerial parts of different varieties of Cichoriumintybus L. J Agric Food Chem 53(16): 6497-6502.

13. Materska M, Perucka I, Stochmal A, Piacente S, Oleszek W (2003) Quantitative and qualitative determination of flavonoids and phenolic acid derivatives from pericarp of hot pepper fruit cv. Bronowicka Ostra. Polish Journal of Food and Nutrition Sciences 12(suppl 2): 72-76.

14. Lu J, Zhenga YL, Luoc L, Wu DM, Suna D, et al. (2006) Quercetin reverses d-galactose induced neurotoxicity in mouse brain. Behav Brain Res 171(2): 251-260.

15. Naidu PS, Kulkarni SK (2004) Quercetin, a bioflavonoid, reverses haloperidol-induced catalepsy. Methods Find Exp Clin Pharmacol 26(5): 323-326.

16. Patil CS, Singh VP, Satyanarayan PSV, Jain NK, Singh A, et al. (2003) Protective Effect of Flavonoids against Aging- and LipopolysaccharideInduced Cognitive Impairment in Mice. Pharmacology 69(2): 59-67.

17. Singh D, Chander V, Chopra K (2004) Quercetin, a Bioflavonoid, Attenuates Ferric Nitrilotriacetate-Induced Oxidative Renal Injury in Rats. Drug Chem Toxicol 27(2): 145-156.

18. Loke WM, Hodgson JM, Proudfoot JM, McKinley JA, Puddey IB, et al. (2008) Pure dietary flavonoids quercetin and (-)-epicatechin augment nitric oxide products and reduce endothelin-1 acutely in healthy men. Am J Clin Nutr 88(4): 1018-1025.

19. Zahedi M, Ghiasvand R, Feizi A, Asgari G, Darvish L (2013) Does Quercetin Improve Cardiovascular Risk factors and Inflammatory Biomarkers in Women with Type 2 Diabetes: A Double-blind Randomized Controlled Clinical Trial. Int J Prev Med 4(7): 777-785.

20. Edwards RL, Lyon T, Litwin SE, Rabovsky A, Symons JD, et al. (2007) Quercetin reduces blood pressure in hypertensive subjects. J Nutr 137(11): 2405-2411.

21. Srivastava S, Ranganatha R, Somasagara, Hegde M, Nishana M, et al (2016) Quercetin, a Natural Flavonoid Interacts with DNA, Arrests Cell Cycle and Causes Tumor Regression by Activating Mitochondrial Pathway of Apoptosis.

22. Shu Y, Liu Y, LiL F, Lou B, Zhou X, et al. (2011) Antibacterial activity of quercetin on oral infectious pathogens. 5(30): 5358-5361.

23. Rivera L, Morón R, Sánchez M, Zarzuelo A, Galisteo M (2008) Quercetin ameliorates metabolic syndrome and improves the inflammatory status in obese Zucker rats. Obesity 16(9): 2081-2087.

24. Kim JH, Kang MJ, Choi HN, Jeong SM, Lee YM, et al. (2011) Quercetin attenuates fasting and postprandial hyperglycemia in animal models of diabetes mellitus. Nutr Res Pract 5(2): 107-111.

25. Torres PM, Ortiz AR, Villalobos MR, Singh N, Medina FJL, et al. (2010) A comparative study of flavonoid analogues on streptozotocin- 
nicotinamide induced diabetic rats: quercetin as a potential antidiabetic agent acting via 11 beta-hydroxysteroid dehydrogenase type 1 inhibition Eur. J Med Chem 45(6): 2606-2612.

26. Dias AS, Porawski M, Alonso M, Marroni N, Pilar S, et al. (2005) Quercetin decreases oxidative stress, NF- $\kappa$ B activation, and iNOS overexpression in liver of streptozotocin-induced diabetic rats. J Nutr 135(10): 2299-2304.
27. Harrison A, Ross Cooper R (2008) Quercetin: health benefits with relevance to TNF- $\alpha$-linked inflammatory diseases. J Pre-clinical \& clinical Res 2(2): 97-101.

28. Townsend EA, Emala CW (2013) Quercetin acutely relaxes airway smooth muscle and potentiates $\beta$-agonist-induced relaxation via dual phosphodiesterase inhibition of PLC $\beta$ and PDE4. Am J Physiol Lung Cell Mol Physiol 305(5): 396-403 